\title{
Lauren Cullen
}

\section{Lost in the Rug: Dismantling the Conference Experience}

\begin{abstract}
Lost in the Rug is an ongoing project and series of workshops responding to Toronto based artist Panya Clark Espinal's participatory artwork Lost in the Wood (2014). This project has developed from an ongoing dialogue and collaboration with the artist's sculptural installation. The series of workshops are organized around the construction of a large-scale hooked rug and the materials and design of the rug have been recycled from Clark Espinal's work.
\end{abstract}

KEYWORDS: Rug Hooking, Workshop, Material Culture, Decolonization, Radical Pedagogy

ox

Lost in the Rug is an ongoing project and series of workshops responding to Toronto based artist Panya Clark Espinal's participatory artwork Lost in the Wood (2014). My project has developed from an ongoing dialogue and collaboration with the artist's sculptural installation. The series of workshops are organized around the construction of a large-scale hooked rug and the materials and design of the rug have been recycled from Clark Espinal's work. ${ }^{1}$

Hooked rugs are historically utilitarian and domestic objects with roots dating back to the mid $18^{\text {th }}$ century in North America. ${ }^{2}$ They are made from recycled materials hooked into the open holes of a loosely woven backing (typically old clothes and feedbags). Group work and the exchange of conversation around handwork is central to the process of rug hooking. ${ }^{3}$ Often accompanied by coffee, bagged lunches, and shared refreshments, these social events are referred to as "gatherings" or "hookins." Rug hooking environments draw people together to work on individual or collective projects, and they continue to remain active sites of making, sharing and lateral exchanges of oral and technical knowledge.

Through the medium and process of rug hooking, the Lost in the Rug workshops investigate collaborative art practice and radical pedagogies within institutional settings. The fifth workshop in the series took place over two days at the Ryerson 
Image Arts Center Student Gallery during the Intersections / Cross-Sections Conference. Attendees were invited to work collectively on a large-scale rug that references a surface of Clark Espinal's multi-media installation and uses re-appropriated scraps of industrial wool from the artwork (Fig.1). Conference attendees signed up for set workshop times and/or dropped into the student gallery throughout the duration of the conference. The workshop's open format facilitated organic conversation and allowed for topics of interest outside of the conventional panel and roundtable format of conferences to be discussed. Unexpected visitors to the gallery provided welcome disruptions, oftentimes contributing to the conversation generated around the table. The gallery setting provided an ideal space to stage an atmosphere akin to an informal rug-hooking gathering, often held in homes or community centers. The rug rested upon a table located in the middle of the room, and eight chairs were set up around it. Documentation from previous workshops was projected on the wall, providing a visual history of the rug's origin. Two corners of the gallery were staged with tools and supplies essential for a hook-in: pre-cut strips of material for hooking and a refreshment station, complete with coffee and snacks. The refreshment stand also featured two of my smaller hooked mats that functioned as productive starting points for conversation throughout the workshop (Fig.2).

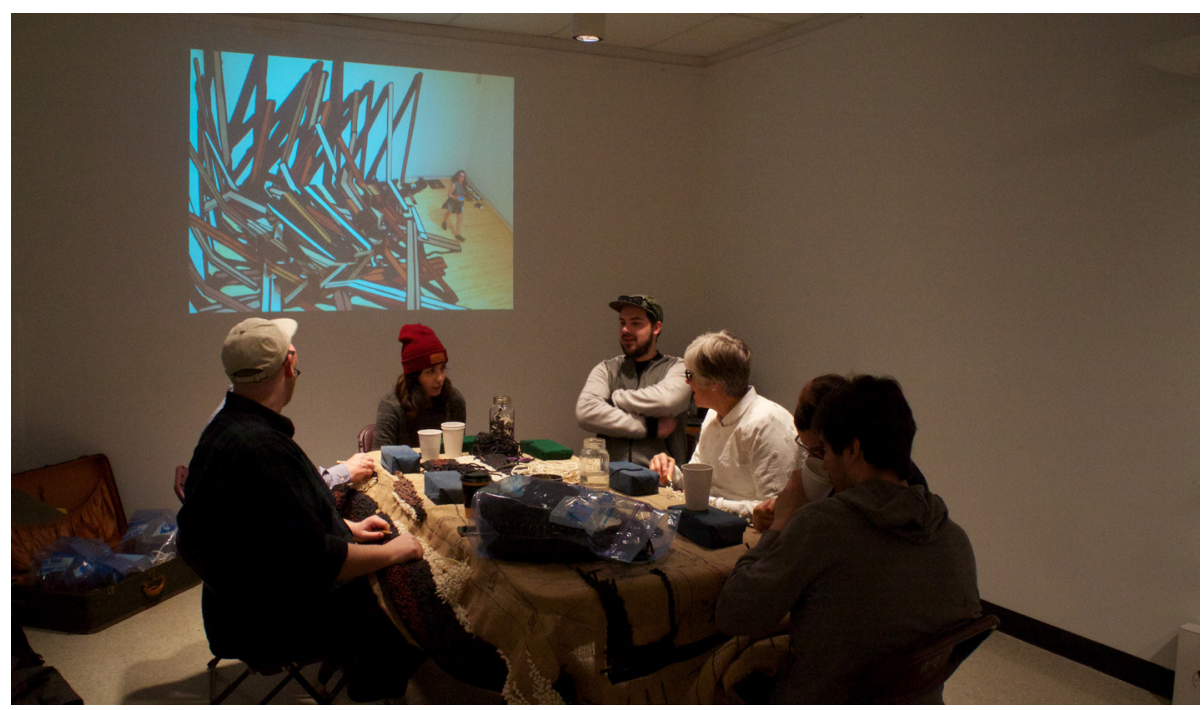

Fig. 1. 


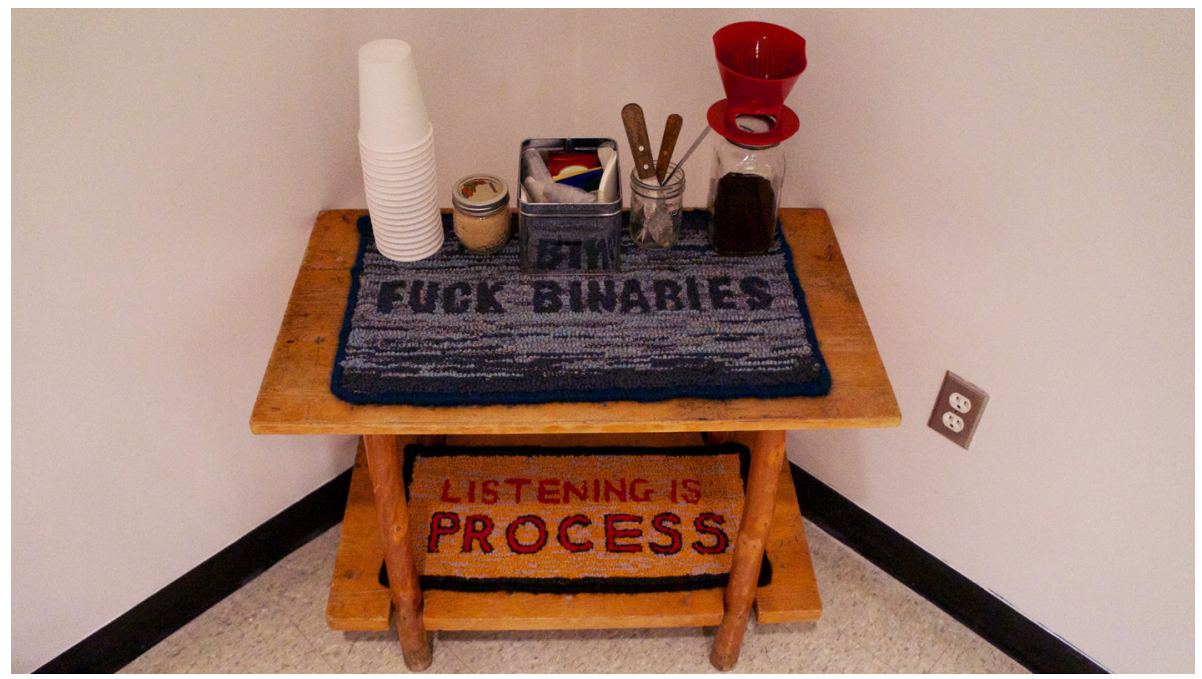

Fig. 2.

Because almost every participant was new to the tools and methods of rug hooking, the nature of the workshop focused on the teaching of techniques. Similar to previous Lost in the Rug workshops, participants shared tips as they learned, assuming the role of teacher upon the arrival of new participants. The social atmosphere in the gallery fostered a space for reciprocal engagement. The practice of sharing hooking techniques extended to conversational exchanges of academic and institutional knowledge; music played in the background and students gathered with peers and teachers. Conversations around the rug and coffee station ebbed and flowed, and included topics of dissertation proposals, questions around program admissions, strategies for self-care as a graduate student, and tactics for challenging dominant and hierarchical forms of knowledge reproduction. At times, the materials served as an illusionary reference point to help explain a theoretical concept to the group (Fig.3).

Often accompanied by coffee, bagged lunches, and shared refreshments, these social events are referred to as "gatherings" or "hook-ins.". Rug hooking environments draw people together to work on individual or collective projects, and they continue to remain active sites of making, sharing and lateral exchanges of oral and technical knowledge. 


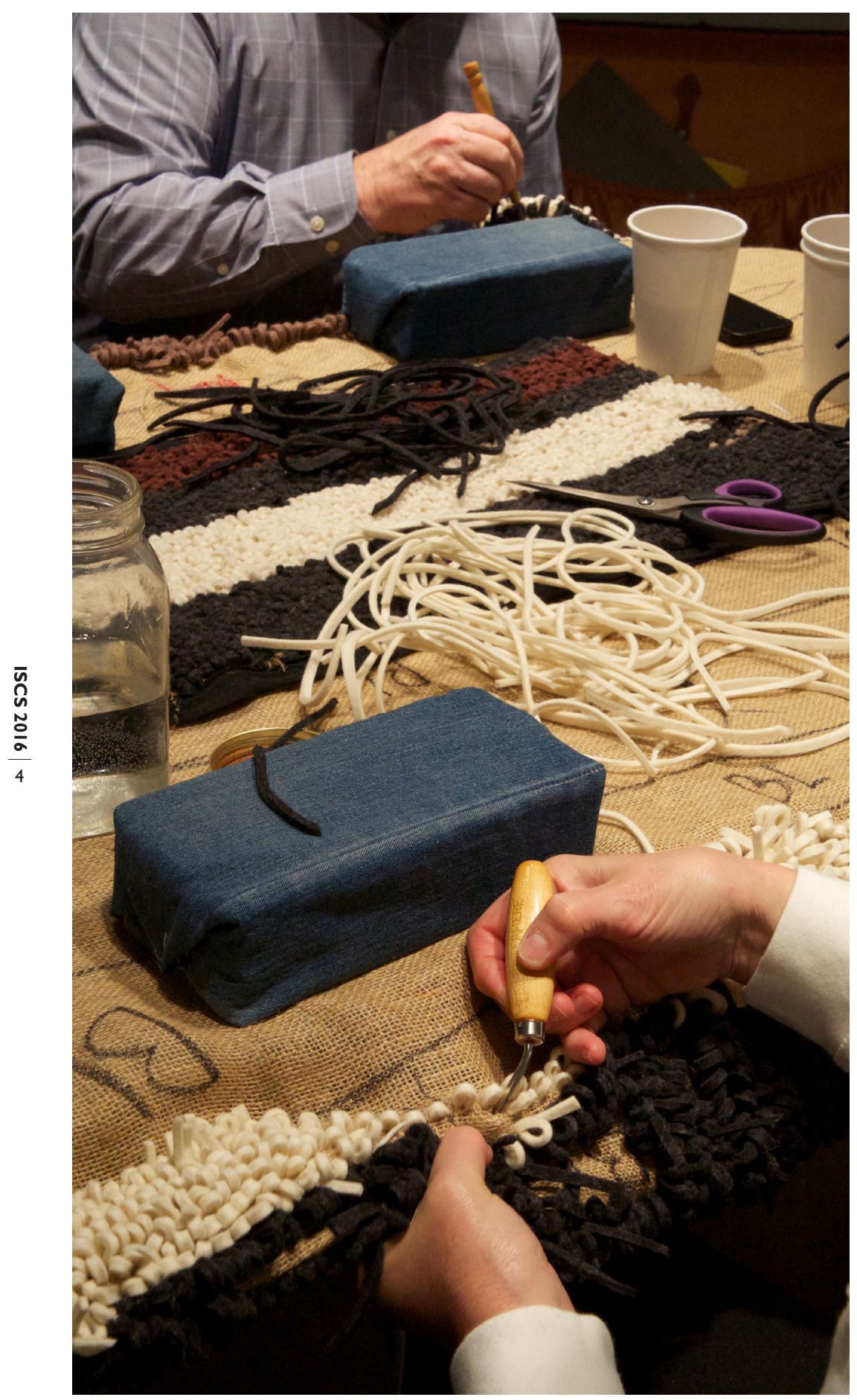

Fig. 3. 
First used to mean "bring together in conversation," the word conference has shifted from its original etymological roots. The academic conference is now commonly understood through the programming of keynotes and thematic panels limited by strict timelines and standardized modes of presentation. While these cornerstones of conferences suggest a well-trodden path for dialogic exchange, they also serve as a prescriptive means of participation. For example, keynotes and panels hosted in classrooms and lecture halls slip into a narrative pattern: one speaking subject engages multiple listening subjects about a niche topic, followed by a structured question-and-answer period. Temporal and spatial restrictions limit socialization and discourage free-form conversation and gossip to occur outside spaces delineated for the delivery of papers and rigorous academic thought. In the context of the Intersections / Cross-Sections conference, hooking served as an alternative means for facilitating conversation. Conference attendees and presenters, teachers and students, makers and novices all worked together on a single task, complementing the sharing network around the table.

The technique and social practice of rug hooking informed crucial aspects to radical pedagogies: the exercise of reflection and observation -- the slow, gradual construction of the rug object mirrored the shared investment of those in conversation around the table. By getting lost in the rug, conference participants practiced a radical approach to communication, mobilized by the desire to share knowledge reciprocally in a safe, accessible space.

\section{Notes}

1. The first four workshops took place at the Carleton University Art Gallery and were held directly in Lost in the Wood. For documentation of Panya Clark Espinal's sculptural installation please visit http://panya.ca/works lost in the wood.php.

2. For a comprehensive article regarding the origins of rug hooking refer to Sharon M.H MacDonald "As the Locusts in Egypt Gathered Crops': Hooked Mat Mania and Cross-Border Shopping in the Early Twentieth Century" in Material History Review 54, no. 3 (2001): 58-70.

3. For a strong example of the relationship to oral history, storytelling and rug hooking please refer to Aen Kroshay Aen Tapee Avec Mi Gineey: Métis Hooked Rugs (2002, Saskatchewan, Canada: Gabriel Dumont Institute), DVD. 